ANNALES

POLONICI MATHEMATICI

$90.1(2007)$

\title{
On the Helmholtz operator of variational calculus in fibered-fibered manifolds
}

\author{
by W. M. Mikulski (Kraków)
}

\begin{abstract}
A fibered-fibered manifold is a surjective fibered submersion $\pi: Y \rightarrow X$ between fibered manifolds. For natural numbers $s \geq r \leq q$ an $(r, s, q)$ th order Lagrangian on a fibered-fibered manifold $\pi: Y \rightarrow X$ is a base-preserving morphism $\lambda$ : $J^{r, s, q} Y \rightarrow \bigwedge^{\operatorname{dim} X} T^{*} X$. For $p=\max (q, s)$ there exists a canonical Euler morphism $\mathcal{E}(\lambda): J^{r+s, 2 s, r+p} Y \rightarrow \mathcal{V}^{*} Y \otimes \bigwedge^{\operatorname{dim} X} T^{*} X$ satisfying a decomposition property similar to the one in the fibered manifold case, and the critical fibered sections $\sigma$ of $Y$ are exactly the solutions of the Euler-Lagrange equation $\mathcal{E}(\lambda) \circ j^{r+s, 2 s, r+p} \sigma=0$. In the present paper, similarly to the fibered manifold case, for any morphism $B: J^{r, s, q} Y \rightarrow$ $\mathcal{V}^{*} Y \otimes \bigwedge^{m} T^{*} X$ over $Y, s \geq r \leq q$, we define canonically a Helmholtz morphism $\mathcal{H}(B): J^{s+p, s+p, 2 p} Y \rightarrow \mathcal{V}^{*} J^{r, s, r} Y \otimes \mathcal{V}^{*} Y \otimes \bigwedge^{\operatorname{dim} X} T^{*} X$, and prove that a morphism $B: J^{r+s, 2 s, r+p} Y \rightarrow \mathcal{V}^{*} Y \otimes \wedge T^{*} M$ over $Y$ is locally variational (i.e. locally of the form $B=\mathcal{E}(\lambda)$ for some $(r, s, p)$ th order Lagrangian $\lambda)$ if and only if $\mathcal{H}(B)=0$, where $p=\max (s, q)$. Next, we study naturality of the Helmholtz morphism $\mathcal{H}(B)$ on fiberedfibered manifolds $Y$ of dimension $\left(m_{1}, m_{2}, n_{1}, n_{2}\right)$. We prove that any natural operator of the Helmholtz morphism type is $c \mathcal{H}(B), c \in \mathbb{R}$, if $n_{2} \geq 2$.
\end{abstract}

0. Introduction. The first problem in variational calculus is to characterize critical values. It is known that the critical sections of a fibered manifold $p: X \rightarrow X_{0}$ with respect to an $r$ th order Lagrangian $\lambda: J^{r} X \rightarrow$ $\bigwedge^{\operatorname{dim} X_{0}} T^{*} X_{0}$ can be characterized as the solutions of the so-called EulerLagrange equation. There exists a unique Euler map $E(\lambda): J^{2 r} X \rightarrow V^{*} X \otimes$ $\bigwedge^{\operatorname{dim} X_{0}} T^{*} X_{0}$ over $X$ satisfying some decomposition formula. Then the Euler-Lagrange equation is $E(\lambda) \circ j^{2 r} \sigma=0$ with unknown section $\sigma$ (see [2]).

The second problem is to characterize morphisms $B: J^{2 r} X \rightarrow$ $V^{*} X \otimes \bigwedge^{\operatorname{dim} X_{0}} T^{*} X_{0}$ over $X$ which are locally variational (i.e. locally of the form $B=E(\lambda)$ for some $r$ th order Lagrangian $\lambda$ ). In [3], for any natural number $r$ and any morphism $B: J^{r} Y \rightarrow V^{*} X \otimes \bigwedge^{\operatorname{dim} X_{0}} T^{*} X_{0}$ over

2000 Mathematics Subject Classification: Primary 58A20.

Key words and phrases: fibered-fibered manifold, $(r, s, q)$-jet prolongation bundle, $(r, s, q)$ th order Lagrangian, Euler morphism, Helmholtz morphism, natural operator. 
$X$ a canonical Helmholtz morphism $H(B): J^{2 r} X \rightarrow V^{*} J^{r} X \otimes V^{*} X \otimes$ $\bigwedge^{\operatorname{dim} X_{0}} T^{*} X_{0}$ over $J^{r} Y$ was described. Next, it was proved that a morphism $B: J^{2 r} X \rightarrow V^{*} X \otimes \bigwedge^{\operatorname{dim} X_{0}} T^{*} X_{0}$ over $X$ is locally variational if and only if $H(B)=0$.

Fibered-fibered manifolds generalize fibered manifolds. They are surjective fibered submersions $\pi: Y \rightarrow X$ between fibered manifolds. They appear naturally in differential geometry if we consider transverse natural bundles (in the sense of R. Wolak [7]) over foliated manifolds (see [5]). A simple example of a fibered-fibered manifold is the following. For any four manifolds $X_{1}, X_{2}, X_{3}, X_{4}$, the obvious projection $\pi: X_{1} \times X_{2} \times X_{3} \times X_{4} \rightarrow X_{1} \times X_{2}$ is a fibered-fibered manifold (we consider $X_{1} \times X_{2} \times X_{3} \times X_{4}$ as the trivial fibered manifold over $X_{1} \times X_{3}$ and $X_{1} \times X_{2}$ as the trivial fibered manifold over $X_{1}$ ). In [5], for fibered-fibered manifolds, using the concept of $(r, s, q)$-jets on fibered manifolds, [2], we extended the notion of $r$-jet prolongation bundle to the $(r, s, q)$-jet prolongation bundle $J^{r, s, q} Y$ for $r, s, q \in \mathbb{N} \backslash\{0\}, s \geq r \leq q$. In [6], we solved the first variational problem for fibered-fibered manifolds. We defined $(r, s, q)$ th order Lagrangians as base preserving (over $X)$ morphisms $\lambda: J^{r, s, q} Y \rightarrow \bigwedge^{\operatorname{dim} X} T^{*} X$. Then similarly to the fibered manifold case we defined critical fibered sections of $Y$. Setting $p=\max (q, s)$ we proved that there exists a canonical Euler morphism $\mathcal{E}(\lambda): J^{r+s, 2 s, r+p} Y$ $\rightarrow \mathcal{V}^{*} Y \otimes \bigwedge^{\operatorname{dim} X} T^{*} X$ of $\lambda$ over $Y$ satisfying a decomposition property similar to the one in the fibered manifold case, where $\mathcal{V} Y \subset T Y$ is the vector subbundle of vectors vertical with respect to two obvious projections from $Y$ (onto $X$ and onto $Y_{0}$ ). Then we deduced that the critical fibered sections $\sigma$ are exactly the solutions of the Euler-Lagrange equation $\mathcal{E}(\lambda) \circ j^{r+s, 2 s, r+p} \sigma=0$. Next, we studied invariance properties of the corresponding Euler operator $\mathcal{E}$. We proved that any natural operator of the Euler morphism type is of the form $c \mathcal{E}$ for some real number $c$. (A similar result for the Euler operator $E$ from variational calculus on fibered manifolds has been obtained by I. Kolář [1].)

The purpose of the present paper is to solve the second problem of variational calculus in fibered-fibered manifolds. Similarly to the fibered manifold case, for any natural numbers $s \geq r \leq q$ and a morphism $B: J^{r, s, q} Y \rightarrow$ $\mathcal{V}^{*} Y \otimes \bigwedge^{\operatorname{dim} X} T^{*} X$ over $Y$ we define canonically a Helmholtz morphism $\mathcal{H}(B): J^{s+p, s+p, 2 p} Y \rightarrow \mathcal{V}^{*} J^{r, s, r} Y \otimes \mathcal{V}^{*} Y \otimes \bigwedge^{\operatorname{dim} X} T^{*} X$ over $J^{r, s, r} Y$, where $p=\max (s, q)$. Then we deduce that a morphism $B: J^{r+s, 2 s, r+p} Y \rightarrow$ $\mathcal{V}^{*} Y \otimes \bigwedge^{\operatorname{dim} X} T^{*} X$ over $Y$ is locally variational (i.e. locally of the form $B=\mathcal{E}(\lambda)$ for some $(r, s, p)$ th order Lagrangian $\lambda)$ if and only if $\mathcal{H}(B)=0$, where $p=\max (s, q)$. Next, we study naturality of the corresponding Helmholtz operator $\mathcal{H}$ on fibered-fibered manifolds $Y$ of (fibered-fibered) dimension $\left(m_{1}, m_{2}, n_{1}, n_{2}\right)$. We prove that any natural operator of the Helmholtz 
operator type is of the form $c \mathcal{H}, c \in \mathbb{R}$, provided $n_{2} \geq 2$. (A similar result for the Helmholtz operator $H$ from variational calculus on fibered manifolds has been obtained by I. Kolár and R. Vitolo [3] for $r=1$ and 2, and by the author [4] for all $r$.)

A 2-fibered manifold is a sequence of two surjective submersions $X \rightarrow$ $X_{1} \rightarrow X_{0}$. For example, given a fibered manifold $X \rightarrow M$ we have the 2-fibered manifolds $T X \rightarrow X \rightarrow M, T^{*} X \rightarrow X \rightarrow M, J^{r} X \rightarrow X \rightarrow M$, etc. Every 2-fibered manifold $X \rightarrow X_{1} \rightarrow X_{0}$ can be considered as a fibered-fibered manifold $X \rightarrow X_{1}$, where we consider $X$ as a fibered manifold $X \rightarrow X_{0}$ and $X_{1}$ as a fibered manifold $X_{1} \rightarrow X_{0}$. So, all our results apply to 2-fibered manifolds.

All manifolds and maps are assumed to be of class $\mathcal{C}^{\infty}$.

\section{Background: variational calculus in fibered manifolds}

1.1. A fibered manifold is a surjective submersion $p: X \rightarrow X_{0}$ between manifolds. If $p^{\prime}: X^{\prime} \rightarrow X_{0}^{\prime}$ is another fibered manifold then a map $f: X \rightarrow X^{\prime}$ is called fibered if there exists a (unique) map $f_{0}: X_{0} \rightarrow X_{0}^{\prime}$ such that $p^{\prime} \circ f=f_{0} \circ p$.

Denote the set of (local) sections of $p$ by $\Gamma X$. The $r$-jet prolongation

$$
J^{r} X=\left\{j_{x_{0}}^{r} \sigma \mid \sigma \in \Gamma X, x_{0} \in X_{0}\right\}
$$

of $X$ is a fibered manifold over $X_{0}$ with respect to the source projection $p^{r}: J^{r} X \rightarrow X_{0}$. If $p^{\prime}: X^{\prime} \rightarrow X_{0}^{\prime}$ is another fibered manifold and $f:$ $X \rightarrow X^{\prime}$ is a fibered map covering a local diffeomorphism $f_{0}: X \rightarrow X_{0}^{\prime}$ then $J^{r} f: J^{r} X \rightarrow J^{r} X^{\prime}$ is given by $J^{r} f\left(j_{x}^{r} \sigma\right)=j_{f_{0}(x)}^{r}\left(f \circ \sigma \circ f_{0}^{-1}\right)$ for $j_{x}^{r} \sigma \in J^{r} X$.

1.2. Let $p: X \rightarrow X_{0}$ be as above. A vector field $V$ on $X$ is projectable if there exists a vector field $V_{0}$ on $X_{0}$ such that $V$ is $p$-related to $V_{0}$. If $V$ is projectable on $X$, then its flow $\operatorname{Exp} t V$ is formed by local fibered diffeomorphisms, and we can define a vector field

$$
\mathcal{J}^{r} V=\frac{\partial}{\partial t}_{\mid t=0} J^{r}(\operatorname{Exp} t V)
$$

on $J^{r} X$. If $V$ is p-vertical (i.e. $V_{0}=0$ ), then $\mathcal{J}^{r} V$ is $p^{r}$-vertical.

1.3. An $r$ th order Lagrangian on a fibered manifold $p: X \rightarrow X_{0}$ with $\operatorname{dim} X_{0}=m$ is a base preserving (over $X_{0}$ ) morphism

$$
\lambda: J^{r} X \rightarrow \bigwedge^{m} T^{*} X_{0}
$$

Given a section $\sigma \in \Gamma X$ and a compact subset $K \subset \operatorname{dom}(\sigma)$ contained in a chart domain, the action is

$$
S(\lambda, \sigma, K)=\int_{K}\left(\lambda \circ j^{r} \sigma\right) .
$$


A section $\sigma \in \Gamma X$ is called critical if for any compact $K \subset \operatorname{dom}(\sigma)$ contained in a chart domain and any $p$-vertical vector field $\eta$ on $X$ with compact support in $p^{-1}(K)$ we have

$$
\left.\frac{d}{d t}\right|_{t=0} S(\lambda, \operatorname{Exp} t \eta \circ \sigma, K)=0 .
$$

By interchanging differentiation and integration we see that $\sigma$ is critical iff for any compact $K$ and $\eta$ as above we have

$$
\int_{K}\left\langle\delta \lambda, \mathcal{J}^{r} \eta\right\rangle \circ j^{r} \sigma=0
$$

where $\delta \lambda: V J^{r} X \rightarrow \wedge^{m} T^{*} X_{0}$ is the $p^{r}$-vertical part of the differential of $\lambda$.

1.4. Given a base preserving morphism $\varphi: J^{q} X \rightarrow \bigwedge^{k} T^{*} X_{0}$, its formal exterior differential $D \varphi: J^{q+1} X \rightarrow \bigwedge^{k+1} T^{*} X_{0}$ is defined by

$$
D \varphi\left(j_{x_{0}}^{q+1} \sigma\right)=d\left(\varphi \circ j^{q} \sigma\right)\left(x_{0}\right)
$$

for every local section $\sigma$ of $X$, where $d$ means the exterior differential at $x_{0} \in X_{0}$ of the local $k$-form $\varphi \circ j^{q} \sigma$ on $X_{0}$.

Further, for every morphism $F: J^{q} X \rightarrow \bigotimes^{l} V^{*} J^{s} X \otimes \bigwedge^{k} T^{*} X_{0}$ over $J^{s} X, s \leq q$, and every $l$-tuple of vertical vector fields $\eta_{1}, \ldots, \eta_{l}$ on $X$, we have the evaluation $F\left(\mathcal{J}^{s} \eta_{1}, \ldots, \mathcal{J}^{s} \eta_{l}\right): J^{q} X \rightarrow \bigwedge^{k} T^{*} X_{0}$. One verifies easily in coordinates that there exists a unique morphism $D F: J^{q+1} X \rightarrow$ $\bigotimes^{l} V^{*} J^{s+1} X \otimes \bigwedge^{k+1} T^{*} X_{0}$ over $J^{s+1} Y$ satisfying

$$
D\left(F\left(\mathcal{J}^{s} \eta_{1}, \ldots, \mathcal{J}^{s} \eta_{l}\right)\right)=(D F)\left(\mathcal{J}^{s+1} \eta_{1}, \ldots, \mathcal{J}^{s+1} \eta_{l}\right)
$$

for all $\eta_{1}, \ldots, \eta_{l}$. It will also be called the formal exterior differential of $F$.

1.5. In the following assertion we do not explicitly indicate the pull-back to $J^{2 r} X$.

Proposition 1 ([3]). For every morphism $B: J^{r} X \rightarrow V^{*} J^{r} X \otimes \bigwedge^{m} T^{*} X_{0}$ over $J^{r} X, m=\operatorname{dim} X_{0}$, there exists a unique pair of morphisms $\mathbf{E}(B): J^{2 r} X \rightarrow V^{*} X \otimes \bigwedge^{m} T^{*} X_{0}, \quad F(B): J^{2 r} X \rightarrow V^{*} J^{r} X \otimes \bigwedge^{m} T^{*} X_{0}$, over $X$ and $J^{r} X$, respectively, such that $B=\mathbf{E}(B)+F(B)$, and $F(B)$ is locally of the form $F(B)=D P$, with $P: J^{2 r-1} X \rightarrow V^{*} J^{r-1} X \otimes \bigwedge^{m-1} T^{*} X_{0}$ over the identity of $J^{r-1} X$.

REMARK 1. If $f: J^{q} X \rightarrow \mathbb{R}$ is a function, we have a coordinate decomposition

$$
D f=\left(D_{i} f\right) d x^{i}
$$

where

$$
D_{i} f=\frac{\partial f}{\partial x^{i}}+\sum_{|\alpha| \leq q} \frac{\partial f}{\partial y_{\alpha}^{p}} y_{\alpha+1_{i}}^{p}: J^{q+1} X \rightarrow \mathbb{R}
$$


is the so-called formal (or total) derivative of $f$ and $\left(x^{i}, y^{k}\right)$ are fiber coordinates on $X$ and $\left(x^{i}, y_{\alpha}^{k}\right)$ are the induced coordinates on $J^{q} X$. The local coordinate form of $\mathbf{E}(B)$ is

$$
\mathbf{E}(B)=\sum_{k=1}^{n} \sum_{|\alpha| \leq r}(-1)^{|\alpha|} D_{\alpha} B_{k}^{\alpha} d y^{k} \otimes d^{m} x
$$

(see [3]), where $d^{m} x=d x^{1} \wedge \cdots \wedge d x^{m}, B=\sum_{k=1}^{n} \sum_{|\alpha| \leq r} B_{k}^{\alpha} d y_{\alpha}^{k} \otimes d^{m} x$ and $D_{\alpha}$ is the iterated formal derivative corresponding to the multiindex $\alpha$.

A morphism $\widetilde{B}: J^{r} X \rightarrow V^{*} X \otimes \bigwedge^{m} T^{*} X_{0}$ over $X$ is called an Euler morphism. The morphism $\mathbf{E}(B)$ is called the formal Euler morphism of $B$.

Let $\lambda: J^{r} X \rightarrow \Lambda^{m} T^{*} X_{0}$ be an $r$ th order Lagrangian. We have $\delta \lambda$ : $J^{r} X \rightarrow V^{*} J^{r} X \otimes \bigwedge^{m} T^{*} X_{0}$ over $J^{r} X$. The morphism $E(\lambda):=\mathbf{E}(\delta \lambda):$ $J^{2 r} X \rightarrow V^{*} X \otimes \bigwedge^{m} T^{*} X_{0}$ over $X$ is called the Euler morphism of $\lambda$.

Proposition 1 and the Stokes theorem immediately yield the following well known fact.

Proposition 2 ([2]). A section $\sigma \in \Gamma X$ is critical iff it satisfies the Euler-Lagrange equation $E(\lambda) \circ j^{2 r} \sigma=0$.

1.6. Let $B: J^{r} X \rightarrow V^{*} X \otimes \wedge T^{*} X_{0}$ be an Euler morphism. We can interpret $B$ as a vertical $\wedge^{m} T^{*} X_{0}$-valued 1-form on $J^{r} X$ by using the canonical projection $V J^{r} X \rightarrow V X$. Then its vertical differential $\delta B$ (defined fiberwise) is a vertical $\wedge^{m} T^{*} X_{0}$-valued 2 -form on $J^{r} X$. For every vertical vector field $\eta$ on $X$, we have $\left\langle\delta B, \mathcal{J}^{r} \eta\right\rangle: J^{r} X \rightarrow V^{*} J^{r} X \otimes \bigwedge^{m} T^{*} X_{0}$. Then we apply the formal Euler operator to obtain $\mathbf{E}\left(\left\langle\delta B, \mathcal{J}^{r} \eta\right\rangle\right): J^{2 r} X \rightarrow V^{*} X \otimes \bigwedge^{m} T^{*} X_{0}$ over $X$.

Proposition 3 ([3]). There exists a unique morphism

$$
H(B): J^{2 r} X \rightarrow V^{*} J^{r} X \otimes V^{*} X \otimes \bigwedge^{m} T^{*} X_{0}
$$

over $J^{r} X$ satisfying

$$
\mathbf{E}\left(\left\langle\delta B, \mathcal{J}^{r} \eta\right\rangle\right)=H(B)\left(\mathcal{J}^{r} \eta\right)
$$

for every vertical vector field $\eta$ on $X$.

REMARK 2. The local coordinate form of $H(B)$ is

$$
H(B)=\sum_{k, l=1}^{n} \sum_{|\alpha| \leq r} H_{k l}^{\alpha} d y_{\alpha}^{k} \otimes d y^{l} \otimes d^{m} x,
$$

where

$$
H_{k l}^{\alpha}=\frac{\partial B_{l}}{\partial y_{\alpha}^{k}}-\sum_{|\beta| \leq r-|\alpha|}(-1)^{|\alpha+\beta|} \frac{(\alpha+\beta) !}{\alpha ! \beta !} D_{\beta} \frac{\partial B_{k}}{\partial y_{\alpha+\beta}^{l}}
$$

and $B=\sum_{k=1}^{n} B_{k} d y^{k} \otimes d^{m} x$ (see [3]). 
The morphism $H(B): J^{2 r} X \rightarrow V^{*} J^{r} X \otimes V^{*} X \otimes \bigwedge^{m} T^{*} X_{0}$ over $J^{r} X$ is called the Helmholtz morphism of $B$.

We have the following characterization of local variationality.

Proposition 4 ([3]). An rth order Euler morphism $B$ is locally variational (i.e. locally of the form $B=E(\lambda)$ for some local rth order Lagrangian $\lambda$ ) if and only if $H(B)=0$.

\section{Variational calculus in fibered-fibered manifolds}

2.1. In [5], we generalized the concept of fibered manifolds as follows. A fibered-fibered manifold is a fibered surjective submersion $\pi: Y \rightarrow X$ between fibered manifolds $p^{Y}: Y \rightarrow Y_{0}$ and $p^{X}: X \rightarrow X_{0}$, i.e. a surjective submersion which sends fibers to fibers such that the restricted maps (between fibers) are submersions. If $\pi^{\prime}: Y^{\prime} \rightarrow X^{\prime}$ is another fibered-fibered manifold then a fibered map $f: Y \rightarrow Y^{\prime}$ is called fibered-fibered if there exists a (unique) fibered map $f_{0}: X \rightarrow X^{\prime}$ such that $\pi^{\prime} \circ f=f_{0} \circ \pi$.

Let $r, s, q \in \mathbb{N} \backslash\{0\}, s \geq r \leq q$.

Denote the set of local fibered maps $\sigma: X \rightarrow Y$ with $\pi \circ \sigma=\operatorname{id}_{\operatorname{dom}(\sigma)}$ (fibered sections) by $\Gamma_{\mathrm{fib}} Y$. By 12.19 in [2], $\sigma, \varrho \in \Gamma_{\mathrm{fib}} Y$ represent the same $(r, s, q)$-jet $j_{x}^{r, s, q} \sigma=j_{x}^{r, s, q} \varrho$ at a point $x \in X$ iff

$$
j_{x}^{r} \sigma=j_{x}^{r} \varrho, \quad j_{x}^{s}\left(\sigma \mid X_{x_{0}}\right)=j_{x}^{s}\left(\varrho \mid X_{x_{0}}\right), \quad j_{x_{0}}^{q} \sigma_{0}=j_{x_{0}}^{q} \varrho_{0},
$$

where $X_{0}$ and $Y_{0}$ are the bases of the fibered manifolds $X$ and $Y, x_{0} \in X_{0}$ is the element under $x, X_{x_{0}}$ is the fiber of $X$ over $x_{0}$, and $\sigma_{0}, \varrho_{0}: X_{0} \rightarrow Y_{0}$ are the underlying maps of $\sigma, \varrho$. The $(r, s, q)$-jet prolongation

$$
J^{r, s, q} Y=\left\{j_{x}^{r, s, q} \sigma \mid \sigma \in \Gamma_{\mathrm{fib}} Y, x \in X\right\}
$$

of $Y$ is a fibered manifold over $X$ with respect to the source projection $\pi_{X}^{r, s, q}: J^{r, s, q} Y \rightarrow X$ (see [4]). We also have the target projection $\pi_{Y}^{r, s, q}$ : $J^{r, s, q} Y \rightarrow Y$. If $\pi^{\prime}: Y^{\prime} \rightarrow X^{\prime}$ is another fibered-fibered manifold and $f:$ $Y \rightarrow Y^{\prime}$ is a fibered-fibered map covering a local fibered diffeomorphism $f_{0}: X \rightarrow X^{\prime}$ then $J^{r, s, q} f: J^{r, s, q} Y \rightarrow J^{r, s, q} Y^{\prime}$ is given by $J^{r, s, q} f\left(j_{x}^{r, s, q} \sigma\right)=$ $j_{f_{0}(x)}^{r, s, q}\left(f \circ \sigma \circ f_{0}^{-1}\right)$ for any $j_{x}^{r, s, q} \sigma \in J^{r, s, q} Y$.

2.2. Let $\pi: Y \rightarrow X$ be a fibered-fibered manifold which is a fibered submersion between fibered manifolds $p^{Y}: Y \rightarrow Y_{0}$ and $p^{X}: X \rightarrow X_{0}$. A projectable vector field $W$ on the fibered manifold $Y$ is projectable-projectable if there exists a $\pi$-related (to $W$ ) projectable vector field $\underline{W}$ on $X$. If $W$ is projectable-projectable on $Y$, then its flow $\operatorname{Exp} t W$ is formed by local fibered-fibered diffeomorphisms, and we define a vector field

$$
\mathcal{J}^{r, s, q} W=\frac{\partial}{\partial t}{ }_{\mid t=0} J^{r, s, q}(\operatorname{Exp} t W)
$$


on $J^{r, s, q} Y$. If additionally $W$ is $\pi$-vertical and $p^{Y}$-vertical (i.e. $W$ is $\pi$ related and $p^{Y}$-related to zero vector fields), then $\mathcal{J}^{r, s, q} W$ is $\pi_{X}^{r, s, q}$-vertical and $p^{Y} \circ \pi_{Y}^{r, s, q}$-vertical.

2.3. Let $r, s, q$ be as above.

An $(r, s, q)$ th order Lagrangian on a fibered-fibered manifold $\pi: Y \rightarrow X$ with $\operatorname{dim} X=m$ is a base preserving (over $X$ ) morphism

$$
\lambda: J^{r, s, q} Y \rightarrow \bigwedge^{m} T^{*} X .
$$

Given a fibered section $\sigma \in \Gamma_{\mathrm{fib}} Y$ and a compact subset $K \subset \operatorname{dom}(\sigma) \subset X$ contained in a chart domain, the action is

$$
S(\lambda, \sigma, K)=\int_{K}\left(\lambda \circ j^{r, s, q} \sigma\right) .
$$

A fibered section $\sigma \in \Gamma_{\mathrm{fib}} Y$ is called critical (with respect to $\lambda$ ) if for any compact $K \subset \operatorname{dom}(\sigma)$ contained in a chart domain and any $\pi$-vertical and $p^{Y}$-vertical vector field $\eta$ on $Y$ with compact support in $\pi^{-1}(K)$ contained in a chart domain we have

$$
\frac{d}{d t}{ }_{\mid t=0} S(\lambda, \operatorname{Exp} t \eta \circ \sigma, K)=0 .
$$

Again we see that $\sigma$ is critical iff for any compact $K$ and $\eta$ as above we have

$$
\int\left\langle\delta \lambda, \mathcal{J}^{r, s, q} \eta\right\rangle j^{r, s, q} \sigma=0,
$$

where $\delta \lambda: \mathcal{V} J^{r, s, q} Y \rightarrow \bigwedge^{m} T^{*} X$ is the restriction of the differential of $\lambda$ to the vector subbundle $\mathcal{V} J^{r, s, q} Y \subset T J^{r, s q} Y$ of vectors vertical with respect to the projections from $J^{r, s, q} Y$ onto $X$ and onto $Y_{0}$.

2.4. Given a base preserving morphism $\varphi: J^{\widetilde{p}, \widetilde{p}, \widetilde{p}} Y \rightarrow \bigwedge^{k} T^{*} X$, its formal exterior differential $\mathcal{D} \varphi: J^{\widetilde{p}+1, \widetilde{p}+1, \widetilde{p}+1} Y \rightarrow \bigwedge^{k+1} T^{*} X$ over $X$ is defined by

$$
\mathcal{D} \varphi\left(j_{x}^{\widetilde{p}+1, \widetilde{p}+1, \widetilde{p}+1} \sigma\right)=d\left(\varphi \circ j^{\widetilde{p}, \widetilde{p}, \widetilde{p}} \sigma\right)(x)
$$

for every local fibered section $\sigma$ of $Y$, where $d$ means the exterior differential at $x \in X$ of the local $k$-form $\varphi \circ j^{\tilde{p}, \tilde{p}, \widetilde{p}} \sigma$ on $X$.

For every morphism $F: J^{\widetilde{p}, \widetilde{p}, \widetilde{p}} Y \rightarrow \bigotimes^{l} \mathcal{V}^{*} J^{\bar{p}, \bar{p}, \bar{p}} Y \otimes \bigwedge^{k} T^{*} X, \bar{p} \leq \widetilde{p}$, over $J^{\bar{p}, \bar{p}, \bar{p}} Y$, and every $l$-tuple of $\pi$-vertical and $p^{Y}$-vertical vector fields $\eta_{1}, \ldots, \eta_{l}$ on $Y$, we have the evaluation $F\left(\mathcal{J}^{\bar{p}, \bar{p}, \bar{p}} \eta_{1}, \ldots, \mathcal{J}^{\bar{p}, \bar{p}, \bar{p}} \eta_{l}\right): J^{\tilde{p}, \widetilde{p}, \widetilde{p}} Y \rightarrow \bigwedge^{k} T^{*} X$. One verifies easily in coordinates that there exists a unique morphism $\mathcal{D} F$ : $J^{\widetilde{p}+1, \widetilde{p}+1, \widetilde{p}+1} Y \rightarrow \bigotimes^{l} \mathcal{V}^{*} J^{\bar{p}+1, \bar{p}+1, \bar{p}+1} Y \otimes \bigwedge^{k+1} T^{*} X$ over $J^{\bar{p}+1, \bar{p}+1, \bar{p}+1} Y$ satisfying

$$
\mathcal{D}\left(F\left(\mathcal{J}^{\bar{p}, \bar{p}, \bar{p}} \eta_{1}, \ldots, \mathcal{J}^{\bar{p}, \bar{p}, \bar{p}} \eta_{l}\right)\right)=(\mathcal{D} F)\left(\mathcal{J}^{\bar{p}+1, \bar{p}+1, \bar{p}+1} \eta_{1}, \ldots, \mathcal{J}^{\bar{p}+1, \bar{p}+1, \bar{p}+1} \eta_{l}\right)
$$

for all $\eta_{1}, \ldots, \eta_{l}$. Here and throughout, $\mathcal{V} J^{\bar{p}, \bar{p}, \bar{p}} Y$ is the vector subbundle of $T J^{\bar{p}, \bar{p}, \bar{p}} Y$ of vectors vertical with respect to the obvious projections from 
$J^{\bar{p}, \bar{p}, \bar{p}} Y$ onto $X$ and onto $Y_{0}$. Also in this case $\mathcal{D} F$ will be called the formal exterior differential of $F$.

2.5. In the following assertion we do not explicitly indicate the pullbacks to $J^{2 p, 2 p, 2 p} Y$ and $J^{p, p, p} Y$.

Proposition 5. Let $r, s, q$ be natural numbers with $s \geq r \leq q$, and set $p=\max (q, s)$. For every morphism $B: J^{r, s, q} Y \rightarrow \mathcal{V}^{*} J^{r, s, q} Y \otimes \bigwedge^{m} T^{*} X$ over $J^{r, s, q} Y$, there is a unique pair of morphisms

$$
\widetilde{\mathbf{E}}(B): J^{2 p, 2 p, 2 p} Y \rightarrow \mathcal{V}^{*} Y \otimes \bigwedge^{m} T^{*} X
$$

and

$$
\mathcal{F}(B): J^{2 p, 2 p, 2 p} Y \rightarrow \mathcal{V}^{*} J^{p, p, p} Y \otimes \bigwedge^{m} T^{*} X,
$$

over $Y$ and $J^{p, p, p} Y$, respectively, such that $B=\widetilde{\mathbf{E}}(B)+\mathcal{F}(B)$, and $\mathcal{F}(B)$ is locally of the form $\mathcal{F}(B)=\mathcal{D} P, P: J^{2 p-1,2 p-1,2 p-1} Y \rightarrow \mathcal{V}^{*} J^{p-1, p-1, p-1} Y$ $\otimes \bigwedge^{m-1} T^{*} X$. Here $\mathcal{V} Y, \mathcal{V} J^{p-1, p-1, p-1} Y$ and $\mathcal{V} J^{p, p, p} Y$ are as in Sections 2.3 and 2.4 .

Proof. Let $\pi_{r, s, q}^{p, p, p}: J^{p, p, p} Y \rightarrow J^{r, s, q} Y$ be the jet projection and let $i_{p}$ : $J^{p, p, p} Y \rightarrow J^{p} Y$ be the canonical inclusion, where in $J^{p} Y$ we consider $Y$ as a fibered manifold over $X$. Using a suitable partition of unity on $X$ and local fibered-fibered coordinate arguments we produce a morphism $\widetilde{B}: J^{p} Y \rightarrow$ $V^{*} J^{p} Y \otimes \bigwedge^{m} T^{*} X$ over $J^{p} Y$ such that $\left(i_{p}\right)^{*} \widetilde{B}=\left(\pi_{r, s, q}^{p, p, p}\right)^{*} B$. Then by the decomposition formula (Proposition 1) there exists a pair of morphisms

$$
\mathbf{E}(\widetilde{B}): J^{2 p} Y \rightarrow V^{*} Y \otimes \bigwedge^{m} T^{*} X, \quad F(\widetilde{B}): J^{2 p} X \rightarrow V^{*} J^{p} Y \otimes \bigwedge^{m} T^{*} X
$$

satisfying $\widetilde{B}=\mathbf{E}(\widetilde{B})+F(\widetilde{B})$, and $F(\widetilde{B})$ is locally of the form $F(\widetilde{B})=D \widetilde{P}$, with $\widetilde{P}: J^{2 p-1} Y \rightarrow V^{*} J^{p-1} Y \otimes \bigwedge^{m-1} T^{*} X$. Taking the pullback $\left(i_{2 p}\right)^{*}$ of both sides of the last formula and using the obvious equality $\mathcal{D}\left(\left(\pi_{r, s, q}^{p, p, p}\right)^{*} B\right)=$ the restriction of $\left(i_{2 p}\right)^{*} D(\widetilde{B})$ to $\mathcal{V} J^{2 p, 2 p, 2 p} Y$, we have the desired decomposition, provided we put $\widetilde{\mathbf{E}}(B)=$ the restriction of $\left(i_{2 p}\right)^{*} \mathbf{E}(\widetilde{B})$ to $\mathcal{V} Y$ and $\mathcal{F}(B)=$ the restriction of $\left(i_{2 p}\right)^{*} F(\widetilde{B})$ to $\mathcal{V} J^{p, p, p} Y$. Since locally $F(\widetilde{B})=D \widetilde{P}$, $\mathcal{F}(B)=\mathcal{D} P$ for $P=$ the restriction of $\left(i_{2 p-1}\right)^{*} \widetilde{P}$ to $\mathcal{V} J^{p-1, p-1, p-1} Y$. Using Remark 1 it is easy to see (see Remark 3 ) that the definition of $\widetilde{\mathbf{E}}(B)$ does not depend on the choice of $\widetilde{B}$.

REMARK 3. Let $\left(x^{i}, X^{I}, y^{k}, Y^{K}\right)$ for $i=1, \ldots, m_{1}, I=1, \ldots, m_{2}$, $k=1, \ldots, n_{1}$ and $K=1, \ldots, n_{2}$ be a fibered-fibered local coordinate system on a fibered-fibered manifold $Y$. For any $f: J^{\widetilde{p}, \widetilde{p}, \widetilde{p}} Y \rightarrow \mathbb{R}$ we have the decomposition

$$
\mathcal{D}(f)=\mathcal{D}_{i}(f) d x^{i}+\mathcal{D}_{I}(f) d X^{I},
$$

where $\mathcal{D}_{i}(f): J^{\widetilde{p}+1, \widetilde{p}+1, \widetilde{p}+1} Y \rightarrow \mathbb{R}$ and $\mathcal{D}_{I}(f): J^{\tilde{p}+1, \widetilde{p}+1, \widetilde{p}+1} Y \rightarrow \mathbb{R}$ are the "total" derivatives of $f$. Let $F: J^{\widetilde{p}} Y \rightarrow \mathbb{R}$ be such that $F \circ i_{\widetilde{p}}=f$. From the 
clear equality $D(F) \circ i_{\widetilde{p}+1}=\mathcal{D}(f)$ we easily deduce that $\mathcal{D}_{i}(f)=D_{i}(F) \circ i_{\widetilde{p}+1}$ and $\mathcal{D}_{I}(f)=D_{I}(F) \circ i_{\widetilde{p}+1}$. In particular, since $D_{i}, D_{I}$ and $D_{i^{\prime}}, D_{I^{\prime}}$ commute, so do $\mathcal{D}_{i}, \mathcal{D}_{I}$ and $\mathcal{D}_{i^{\prime}}, \mathcal{D}_{I^{\prime}}$. From the formulas for $D_{i}$ and $D_{I}$ (see Remark 1 ) and from the above formulas for $\mathcal{D}_{i}$ and $\mathcal{D}_{I}$ we easily see that in local coordinates

$$
\mathcal{D}_{i}(f)=\frac{\partial f}{\partial x^{i}}+\sum_{k=1}^{n_{1}} \sum_{|\widetilde{\alpha}| \leq \widetilde{p}} \frac{\partial f}{\partial y_{\widetilde{\alpha}}^{k}} y_{\widetilde{\alpha}+1_{i}}^{k}+\sum_{K=1}^{n_{2}} \sum_{|\widetilde{\beta}|+|\widetilde{\gamma}| \leq \widetilde{p}} \frac{\partial f}{\partial Y_{(\widetilde{\beta}, \widetilde{\gamma}}^{K}} Y_{\left(\widetilde{\beta}+1_{i}, \widetilde{\gamma}\right)}^{K}
$$

and

$$
\mathcal{D}_{I}(f)=\frac{\partial f}{\partial X^{I}}+\sum_{K=1}^{n_{2}} \sum_{|\widetilde{\beta}|+|\widetilde{\gamma}| \leq \widetilde{p}} \frac{\partial f}{\partial Y_{(\widetilde{\beta}, \widetilde{\gamma})}^{K}} Y_{\left(\widetilde{\beta}, \widetilde{\gamma}+1_{I}\right)}^{K},
$$

where $\left(x^{i}, X^{I}, y_{\widetilde{\alpha}}^{k}, Y_{(\widetilde{\beta}, \widetilde{\gamma})}^{K}\right)$ is the induced coordinate system on $J^{\widetilde{p}, \widetilde{p}, \widetilde{p}} Y, \widetilde{\alpha}=$ $\left(\widetilde{\alpha}^{1}, \ldots, \widetilde{\alpha}^{m_{1}}\right), \widetilde{\beta}=\left(\widetilde{\beta}^{m_{1}}, \ldots, \widetilde{\beta}^{m_{1}}\right)$ and $\widetilde{\gamma}=\left(\widetilde{\gamma}^{1}, \ldots, \widetilde{\gamma}^{m_{2}}\right)$.

Let $\left(x^{i}, X^{I}, y_{\alpha}^{k}, Y_{(\beta, \gamma)}^{K}\right)$ be the induced coordinates on $J^{p, p, p} Y$, where $p=$ $\max (s, q)$. Then using the formula in Remark 1 it is easy to see that the local coordinate form of $\widetilde{\mathbf{E}}(B)$ is

$$
\widetilde{\mathbf{E}}(B)=\sum_{K=1}^{n_{2}} \sum_{|\beta|+|\gamma| \leq p}(-1)^{|\beta|+|\gamma|} \mathcal{D}_{(\beta, \gamma)} B_{K}^{(\beta, \gamma)} d Y^{K} \otimes\left(d^{m_{1}} x \wedge d^{m_{2}} X\right),
$$

where $d^{m_{1}} x=d x^{1} \wedge \cdots \wedge d x^{m_{1}}, d^{m_{2}} X=d X^{1} \wedge \cdots \wedge d X^{m_{2}},\left(\pi_{r, s, q}^{p, p, p}\right)^{*} B=$ $\sum_{K=1}^{n_{2}} \sum_{|\beta|+|\gamma| \leq p} B_{K}^{(\beta, \gamma)} d_{(\beta, \gamma)}^{K} \otimes\left(d^{m_{1}} \wedge d^{m_{2}} X\right)$ and $\mathcal{D}_{(\beta, \gamma)}$ denotes the iterated "total" derivative with $\beta=\left(\beta^{1}, \ldots, \beta^{m_{1}}\right), \gamma=\left(\gamma^{1}, \ldots, \gamma^{m_{2}}\right)$.

From the above local formula it follows that $\widetilde{\mathbf{E}}(B)$ can be factorized through $J^{r+s, 2 s, r+p} Y, p=\max (s, q)$.

A morphism $\widetilde{B}: J^{r, s, q} Y \rightarrow \mathcal{V}^{*} Y \otimes \bigwedge^{m} T^{*} X$ over $Y$ is called an Euler morphism. The morphism $\widetilde{\mathbf{E}}(B): J^{2 p, 2 p, 2 p} Y \rightarrow \mathcal{V}^{*} Y \otimes \bigwedge^{m} T^{*} X$ over $Y$ is called the formal Euler morphism of $B$.

Let $\lambda$ be an $(r, s, q)$ th order Lagrangian on $Y$, and $p=\max (s, q)$. We have $\delta \lambda: J^{r, s, q} Y \rightarrow \mathcal{V}^{*} J^{r, s, q} Y \otimes \bigwedge^{m} T^{*} X$. The morphism $\mathcal{E}(\lambda)=\widetilde{\mathbf{E}}(\delta \lambda)$ : $J^{2 p, 2 p, 2 p} Y \rightarrow \mathcal{V}^{*} Y \otimes \bigwedge^{m} T^{*} X$ over $Y$ is called the Euler morphism of $\lambda$.

By the above-mentioned property of $\widetilde{\mathbf{E}}(B)$ it follows that $\mathcal{E}(\lambda)$ can also be factorized through $J^{r+s, 2 s, r+p} Y$.

Proposition 5 and the Stokes theorem yield the following fact.

Proposition 6 ([6]). A fibered section $\sigma \in \Gamma_{\mathrm{fib}} Y$ is critical iff it satisfies the Euler-Lagrange equation $\mathcal{E}(\lambda) \circ j^{2 p, 2 p, 2 p} \sigma=0$. By the above-mentioned property of $\mathcal{E}(\lambda)$ this equation is $\mathcal{E}(\lambda) \circ j^{r+s, 2 s, r+p} \sigma=0$. 
2.6. Let $B: J^{r, s, q} Y \rightarrow \mathcal{V}^{*} Y \otimes \bigwedge^{m} T^{*} X$ be an Euler morphism, and $p=\max (s, q)$. Using the canonical projections $\mathcal{V}^{*} J^{r, s, q} Y \rightarrow \mathcal{V} Y$, we can interpret $B$ as a vertical $\bigwedge^{m} T^{*} X$-valued 1 -form on $J^{r, s, q} Y$. Then the vertical differential $\delta B$ (defined fiberwise) is a vertical $\bigwedge^{m} T^{*} X$-valued 2-form on $J^{r, s, q} Y$. For every $\pi$-vertical and $p^{Y}$-vertical vector field $\eta$ on $Y$, we have $\left\langle\delta B, \mathcal{J}^{r, s, q} \eta\right\rangle: J^{r, s, q} Y \rightarrow \mathcal{V}^{*} J^{r, s, q} Y \otimes \bigwedge^{m} T^{*} X$ over $J^{r, s, q} Y$. Then we can apply the formal Euler operator to obtain $\widetilde{\mathbf{E}}\left(\left\langle\delta B, \mathcal{J}^{r, s, q} \eta\right\rangle\right): J^{2 p, 2 p, 2 p} Y \rightarrow$ $\mathcal{V}^{*} Y \otimes \bigwedge^{m} T^{*} X$ over $Y$.

Proposition 7. There exists a unique morphism

$$
\mathcal{H}(B): J^{2 p, 2 p, 2 p} Y \rightarrow \mathcal{V}^{*} J^{p, p, p} Y \otimes \mathcal{V}^{*} Y \otimes \bigwedge^{m} T^{*} X
$$

over $J^{p, p, p} Y$ satisfying

$$
\widetilde{\mathbf{E}}\left(\left\langle\delta B, \mathcal{J}^{r, s, q} \eta\right\rangle\right)=\mathcal{H}(B)\left(\mathcal{J}^{p, p, p} \eta\right)
$$

for every $\pi$-vertical and $p^{Y}$-vertical vector field $\eta$ on $Y$.

Proof. That $\mathcal{H}(B)$ is unique is clear. We prove the existence.

As in the proof of Proposition 5, we have a morphism $\widetilde{B}: J^{p} Y \rightarrow$ $V^{*} Y \otimes \bigwedge^{m} T^{*} X$ over $Y$ such that $\left(\pi_{r, s, q}^{p, p, p}\right)^{*} B=$ the restriction of $\left(i_{p}\right)^{*} \widetilde{B}$ to $\mathcal{V}^{*} Y$. Then by Proposition $3, \mathbf{E}\left(\left\langle\delta \widetilde{B}, \mathcal{J}^{p} \eta\right\rangle\right)=H(\widetilde{B})\left(\mathcal{J}^{p} \eta\right)$, where $H(\widetilde{B})$ is the Helmholtz morphism of $\widetilde{B}$. Applying the pull-back $\left(i_{2 p}\right)^{*}$ to both sides of the last equality and using the definition of $\widetilde{\mathbf{E}}\left(\left\langle\delta B, \mathcal{J}^{r, s, q} \eta\right\rangle\right)$ (see the proof of Proposition 5) we obtain the desired equality for $\mathcal{H}(B)=$ the restriction of $\left(i_{2 p}\right)^{*} H(\widetilde{B})$ to $\mathcal{V} J^{p, p, p} Y \times_{Y} \mathcal{V} Y$. One can show (see Remark 4 below) that the definition of $\mathcal{H}(B)$ is independent of the choice of $\widetilde{B}$.

REMARK 4. It follows from the formula in Remark 2 and from the definition of $\mathcal{H}(B)$ in the proof of Proposition 7 that the local coordinate form of $\mathcal{H}(B)$ is

$$
\mathcal{H}(B)=\sum_{K, L=1}^{n_{2}} \sum_{|\beta|+|\gamma| \leq p} \mathcal{H}_{K L}^{(\beta, \gamma)} d Y_{(\beta, \gamma)}^{K} \otimes d Y^{L} \otimes d^{m_{1}} x \otimes d^{m_{2}} X,
$$

where

$$
\begin{aligned}
\mathcal{H}_{K L}^{(\beta, \gamma)=} & \frac{\partial B_{L}}{\partial Y_{(\beta, \gamma)}^{K}} \\
& -\sum_{|\widetilde{\beta}|+|\widetilde{\gamma}| \leq p-|\beta|-|\gamma|}(-1)^{|\widetilde{\beta}|+|\widetilde{\gamma}|} \frac{(\beta+\widetilde{\beta}) !(\gamma+\widetilde{\gamma}) !}{\beta ! \widetilde{\beta} ! \gamma ! \widetilde{\gamma} !} \mathcal{D}_{(\widetilde{\beta}, \widetilde{\gamma})} \frac{\partial B_{K}}{\partial Y_{(\beta+\widetilde{\beta}, \gamma+\widetilde{\gamma})}^{L}}
\end{aligned}
$$

and $B=\sum_{K=1}^{n_{1}} B_{K} d Y^{K} \otimes d^{m_{1}} x \wedge d^{m_{2}} X$.

From this local formula it follows easily that $\mathcal{H}(B)$ can be factorized through $\left(J^{s+p, s+p, 2 p} Y \times_{J^{r, s, r} Y} \mathcal{V} J^{r, s, r} Y\right) \times_{Y} \mathcal{V} Y$. 
We have the following characterization of local variationality.

Proposition 8. Let $s \geq r \leq q$ be natural numbers and $p=\max (s, q)$. $A(2 p, 2 p, 2 p)$ th order Euler morphism $B$ is locally variational (i.e. locally of the form $\mathcal{E}(\lambda)$ for some $(p, p, p)$ th order Lagrangian $\lambda)$ if and only if $\mathcal{H}(B)=0$.

Moreover, if a $(2 p, 2 p, 2 p)$ th order Euler morphism $B$ is locally variational and factorizes through $J^{r+s, 2 s, r+p} Y$, then locally $B=\mathcal{E}(\lambda)$ for some $(r, s, p)$ th order Lagrangian.

Proof. Suppose locally $B=\mathcal{E}(\lambda)$. Choose a local $p$ th order Lagrangian $\Lambda: J^{p} Y \rightarrow \bigwedge^{m} T^{*} X$ such that $\lambda \circ \pi_{r, s, q}^{p, p, p}=\left(i_{p}\right)^{*} \Lambda$. We see that $\delta \lambda$ is the restriction of $\left(i_{p}\right)^{*} \delta \Lambda$ to $\mathcal{V} Y$. Hence $\mathcal{H}(B)=\mathcal{H}(\mathcal{E}(\lambda))$ is the restriction of $\left(i_{4 p}\right)^{*} H(E(\Lambda))$ to $\mathcal{V} J^{2 p} Y \times_{Y} \mathcal{V} Y$. Since $H(E(\Lambda))=0$ (see Proposition 4), also $\mathcal{H}(B)=0$.

To prove the converse we choose local fibered-fibered coordinates $\left(x^{i}, X^{I}, y^{k}, Y^{K}\right)$ on $U \subset Y$. In this coordinate system we have the obvious projection $\Pi: J^{\widetilde{p}} U=\mathbb{R}^{M} \rightarrow J^{\widetilde{p}, \widetilde{p}, \widetilde{p}} U=\mathbb{R}^{N}$ for any $\widetilde{p}$. Let $\mathcal{H}(B)=0$. Then (using the local formula) we have $H\left(\Pi^{*} B\right)=0$. Proposition 4 yields $\Pi^{*} B=E(\Lambda)$ for some $p$ th order Lagrangian $\Lambda$ on $U$. Thus $B=\mathcal{E}(\lambda)$ for $\lambda=\left(i_{p}\right)^{*} \Lambda$.

The "moreover" part can be deduced in the following way. By the assumption, there is $\tilde{\lambda}$ of order $(p, p, p)$ such that $B=\mathcal{E}(\widetilde{\lambda})$ over $U$, where $\left(U, x^{i}, X^{I}, y^{k}, Y^{K}\right)$ are fibered-fibered coordinates. Using these coordinates we can consider the obvious inclusion $J: J^{r, s, p} U=\mathbb{R}^{M} \rightarrow J^{p, p, p} U=\mathbb{R}^{N}$, $J(v)=(v, 0)$. Then (using the local expression of $\widetilde{\mathbf{E}}(\delta \lambda))$ we see that $B=\mathcal{E}\left(J^{*} \widetilde{\lambda}\right)$.

3. On naturality of the Helmholtz operator. We say that a fibered manifold $p: X \rightarrow X_{0}$ is of dimension $(m, n)$ if $\operatorname{dim} X_{0}=m$ and $\operatorname{dim} X=$ $m+n$. All $(m, n)$-dimensional fibered manifolds and their local fibered diffeomorphisms form a category which we denote by $\mathcal{F} \mathcal{M}_{m, n}$ and which is local and admissible in the sense of [2].

Similarly, a fibered-fibered manifold $\pi: Y \rightarrow X$ is of dimension $\left(m_{1}, m_{2}\right.$, $\left.n_{1}, n_{2}\right)$ if the fibered manifold $X$ is of dimension $\left(m_{1}, n_{1}\right)$ and the fibered manifold $Y$ is of dimension $\left(m_{1}+n_{1}, m_{2}+n_{2}\right)$. All $\left(m_{1}, m_{2}, n_{1}, n_{2}\right)$-dimensional fibered-fibered manifolds and their fibered-fibered local diffeomorphisms form a category which we denote by $\mathcal{F} \mathcal{M}_{m_{1}, m_{2}, n_{1}, n_{2}}$ and which is local and admissible in the sense of [2]. The standard $\left(m_{1}, m_{2}, n_{1}, n_{2}\right)$ dimensional trivial fibered-fibered manifold $\pi: \mathbb{R}^{m_{1}} \times \mathbb{R}^{m_{2}} \times \mathbb{R}^{n_{1}} \times \mathbb{R}^{n_{2}} \rightarrow$ $\mathbb{R}^{m_{1}} \times \mathbb{R}^{m_{2}}$ will be denoted by $\mathbb{R}^{m_{1}, m_{2}, n_{1}, n_{2}}$. Any $\left(m_{1}, m_{2}, n_{1}, n_{2}\right)$-dimensional fibered-fibered manifold is locally $\mathcal{F M}_{m_{1}, m_{2}, n_{1}, n_{2}}$-isomorphic to the standard $\mathcal{F} \mathcal{M}_{m_{1}, m_{2}, n_{1}, n_{2}}$-object $\mathbb{R}^{m_{1}, m_{2}, n_{1}, n_{2}}$. 
Given two fibered manifolds $Z_{1} \rightarrow M$ and $Z_{2} \rightarrow M$ over the same base $M$, we denote the space of all base preserving fibered manifold morphisms of $Z_{1}$ into $Z_{2}$ by $\mathcal{C}_{M}^{\infty}\left(Z_{1}, Z_{2}\right)$. In [3], [4], the authors studied the $r$ th order Helmholtz morphism $H(B)$ of variational calculus on an $(m, n)$-dimensional fibered manifold $p: X \rightarrow X_{0}$ as the Helmholtz operator

$H: \mathcal{C}_{X}^{\infty}\left(J^{r} X, V^{*} X \otimes \bigwedge^{m} T^{*} X_{0}\right) \rightarrow \mathcal{C}_{J^{r} X}^{\infty}\left(J^{2 r} X, V^{*} J^{r} X \otimes V^{*} X \otimes \bigwedge^{m} T^{*} X_{0}\right)$.

They deduced the following classification theorem:

Theorem 1 ([3], [4]). Any $\mathcal{F} \mathcal{M}_{m, n}$-natural operator (in the sense of [2]) of the type of the Helmholtz operator is of the form $\mathrm{cH}, c \in \mathbb{R}$, provided $n \geq 2$.

The purpose of the present section is to obtain a similar result in the fibered-fibered manifold case. Namely, we study the Helmholtz morphism $\mathcal{H}(B)$ of variational calculus on an $\left(m_{1}, m_{2}, n_{1}, n_{2}\right)$-dimensional fiberedfibered manifold $\pi: Y \rightarrow X$ as the Helmholtz operator

$$
\begin{aligned}
\mathcal{H}: \mathcal{C}_{Y}^{\infty}\left(J^{r, s, q} Y\right. & \left.\mathcal{V}^{*} Y \otimes \bigwedge^{m} T^{*} X\right) \\
& \rightarrow \mathcal{C}_{J^{p, p, p} Y}^{\infty}\left(J^{2 p, 2 p, 2 p} Y, \mathcal{V}^{*} J^{p, p, p} Y \otimes \mathcal{V}^{*} Y \otimes \bigwedge^{m} T^{*} X\right),
\end{aligned}
$$

where $s \geq r \leq q$ are natural numbers, $p=\max (s, q)$ and $m=m_{1}+m_{2}$ $=\operatorname{dim} X$. We prove the following classification theorem.

THEOREM 2. Any $\mathcal{F M}_{m_{1}, m_{2}, n_{1}, n_{2}}$-natural operator (in the sense of [2]) of the type of the Helmholtz operator is of the form $c \mathcal{H}, c \in \mathbb{R}$, provided $n_{2} \geq 2$.

REMARK 5. In view of Remark 3 the assertion of Theorem 2 also holds for natural operators

$$
\begin{aligned}
D: \mathcal{C}_{Y}^{\infty}\left(J^{r, s, q} Y, \mathcal{V}^{*} Y \otimes \bigwedge^{m} T^{*} X\right) & \\
& \rightarrow \mathcal{C}_{J^{r, s, r} Y}^{\infty}\left(J^{s+p, s+p, 2 p} Y, \mathcal{V}^{*} J^{r, s, r} Y \otimes \mathcal{V}^{*} Y \otimes \bigwedge^{m} T^{*} X\right) .
\end{aligned}
$$

REMARK 6. The assumption of the last theorem means that for any $\mathcal{F} \mathcal{M}_{m_{1}, m_{2}, n_{1}, n_{2}}$-morphism $f: Y \rightarrow Y^{\prime}$ and any morphisms

$$
B \in \mathcal{C}_{Y}^{\infty}\left(J^{r, s, q} Y, \mathcal{V}^{*} Y \otimes \bigwedge^{m} T^{*} X\right)
$$

and

$$
B^{\prime} \in \mathcal{C}_{Y^{\prime}}^{\infty}\left(J^{r, s, q} Y^{\prime}, \mathcal{V}^{*} Y^{\prime} \otimes \bigwedge^{m} T^{*} X^{\prime}\right),
$$

if $B$ and $B^{\prime}$ are $f$-related then so are $D(B)$ and $D\left(B^{\prime}\right)$. Moreover $D$ is regular and local. The regularity means that $D$ transforms a smoothly parametrized family of appriopriate type morphisms into a smoothly parametrized family of appriopriate type morphisms. The locality means that $D(B)_{u}$ depends on the germ of $B$ at $\pi_{r, s, q}^{p, p, p}(u)$. 
Proof of Theorem 2. Let $D$ be an operator in question.

Let $\left(x^{i}, X^{I}, y^{k}, Y^{K}\right)$ be the usual fibered-fibered coordinate system on $\mathbb{R}^{m_{1}, m_{2}, n_{1}, n_{2}}, i=1, \ldots, m_{1}, I=1, \ldots, m_{2}, k=1, \ldots, n_{1}, K=1, \ldots, n_{2}$.

Since an $\mathcal{F M}_{m_{1}, m_{2}, n_{1}, n_{2}}$-map

$$
\left(x^{i}, X^{I}, y^{k}-\sigma^{k}\left(x^{i}, X^{I}\right), Y^{K}-\Sigma^{K}\left(x^{i}, X^{I}\right)\right)
$$

sends $j_{(0,0)}^{2 p, 2 p, 2 p}\left(x^{i}, X^{I}, \sigma^{k}, \Sigma^{K}\right)$ to

$$
\Theta=j_{(0,0)}^{2 p, 2 p, 2 p}\left(x^{i}, X^{I}, 0,0\right) \in\left(J^{2 p, 2 p, 2 p}\left(\mathbb{R}^{m_{1}, m_{2}, n_{1}, n_{2}}\right)\right)_{(0,0,0,0)},
$$

$J^{2 p, 2 p, 2 p}\left(\mathbb{R}^{m_{1}, m_{2}, n_{1}, n_{2}}\right)$ is the $\mathcal{F} \mathcal{M}_{m_{1}, m_{2}, n_{1}, n_{2}}$-orbit of $\Theta$. Then $D$ is uniquely determined by the evaluations

$$
\left\langle D(B)_{\Theta}, w \otimes v\right\rangle \in \bigwedge^{m} T_{0}^{*} \mathbb{R}^{m}
$$

for all

$$
B \in \mathcal{C}_{\mathbb{R}^{m_{1}, m_{2}, n_{1}, n_{2}}}^{\infty}\left(J^{r, s, q}\left(\mathbb{R}^{m_{1}, m_{2}, n_{1}, n_{2}}\right), \mathcal{V}^{*} \mathbb{R}^{m_{1}, m_{2}, n_{1}, n_{2}} \otimes \bigwedge^{m} T^{*} \mathbb{R}^{m}\right),
$$
$w \in \mathcal{V}_{\pi_{p, p, p}^{2 p, 2 p, 2 p}(\Theta)} J^{p, p, p}\left(\mathbb{R}^{m_{1}, m_{2}, n_{1}, n_{2}}\right), \quad v \in T_{0} \mathbb{R}^{n_{2}}=\mathcal{V}_{(0,0,0,0)} \mathbb{R}^{m_{1}, m_{2}, n_{1}, n_{2}}$.

Using the invariance of $D$ with respect to $\mathcal{F} \mathcal{M}_{m_{1} m_{2}, n_{1}, n_{2}}$-maps of the form $\operatorname{id}_{\mathbb{R}^{m}} \times \psi$ for appriopriate linear $\psi$ (since $n_{2} \geq 2$ ) we find that $D$ is uniquely determined by the evaluations

$$
\left\langle D(B)_{\Theta}, \frac{d}{d t_{0}}\left(t j_{(0,0)}^{p, p, p}\left(x^{i}, X^{I}, 0, \ldots, 0, f\left(x^{i}, X^{I}\right), 0, \ldots, 0\right)\right) \otimes \frac{\partial}{\partial Y^{2}}{ }_{0}\right\rangle
$$

for all

$$
B \in \mathcal{C}_{\mathbb{R}^{m_{1}, m_{2}, n_{1}, n_{2}}}^{\infty}\left(J^{r, s, q}\left(\mathbb{R}^{m_{1}, m_{2}, n_{1}, n_{2}}\right), \mathcal{V}^{*} \mathbb{R}^{m_{1}, m_{2}, n_{1}, n_{2}} \otimes \bigwedge^{m} T^{*} \mathbb{R}^{m}\right)
$$

and all $f: \mathbb{R}^{m} \rightarrow \mathbb{R}$, where $f\left(x^{i}, X^{I}\right)$ is at position $Y^{1}$.

Using the invariance of $D$ with respect to the $\mathcal{F} \mathcal{M}_{m_{1} m_{2}, n_{1}, n_{2}}$-map

$$
\left(x^{1}, \ldots, x^{m_{1}}, X^{1}, \ldots, X^{m_{2}}, y^{1}, \ldots, y^{n_{1}}, Y^{1}+f\left(x^{i}, X^{I}\right) Y^{1}, Y^{2}, \ldots, Y^{n_{2}}\right)
$$

preserving $\Theta$ we can assume $f=1$, i.e. $D$ is uniquely determined by the evaluations

$$
\left\langle D(B)_{\Theta}, \frac{d}{d t_{0}}\left(t j_{(0,0)}^{p, p, p}\left(x^{i}, X^{I}, 0, \ldots, 0,1,0, \ldots, 0\right)\right) \otimes \frac{\partial}{\partial Y^{2}}\right\rangle \in \bigwedge^{m} T_{0}^{*} \mathbb{R}^{m}
$$

for all

$$
B \in \mathcal{C}_{\mathbb{R}^{m_{1}, m_{2}, n_{1}, n_{2}}}^{\infty}\left(J^{r, s, q}\left(\mathbb{R}^{m_{1}, m_{2}, n_{1}, n_{2}}\right), \mathcal{V}^{*} \mathbb{R}^{m_{1}, m_{2}, n_{1}, n_{2}} \otimes \bigwedge^{m} T^{*} \mathbb{R}^{m}\right),
$$

where 1 is at position $Y^{1}$.

Consider a morphism

$$
B \in \mathcal{C}_{\mathbb{R}^{m_{1}, m_{2}, n_{1}, n_{2}}}\left(J^{r, s, q}\left(\mathbb{R}^{m_{1}, m_{2}, n_{1}, n_{2}}\right), \mathcal{V}^{*} \mathbb{R}^{m_{1}, m_{2}, n_{1}, n_{2}} \otimes \bigwedge^{m} T^{*} \mathbb{R}^{m}\right) .
$$


Using the invariance of $D$ with respect to the $\mathcal{F} \mathcal{M}_{m_{1}, m_{2}, n_{1}, n_{2}}$-maps

$$
\psi_{\tau, \mathcal{T}}=\left(x^{i}, X^{I}, \frac{1}{\tau^{k}} y^{k}, \frac{1}{\mathcal{T}^{K}} Y^{K}\right)
$$

for $\tau^{k} \neq 0$ and $\tau^{K} \neq 0$ we get the homogeneity condition

$$
\begin{aligned}
& \left\langle D\left(\left(\psi_{\tau, \mathcal{T}}\right)_{*} B\right)_{\Theta}, \frac{d}{d t_{0}}\left(t_{(0,0)}^{p, p, p}\left(x^{i}, X^{I}, 0, \ldots, 0,1,0, \ldots, 0\right)\right) \otimes \frac{\partial}{\partial Y^{2}}{ }_{0}\right\rangle \\
& =\mathcal{T}^{1} \mathcal{T}^{2}\left\langle D(B)_{\Theta}, \frac{d}{d t_{0}}\left(t j_{(0,0)}^{p, p, p}\left(x^{i}, X^{I}, 0, \ldots, 0,1,0, \ldots, 0\right)\right) \otimes \frac{\partial}{\partial Y^{2}}{ }_{0}\right\rangle
\end{aligned}
$$

for $\tau=\left(\tau^{k}\right)$ and $\mathcal{T}=\left(\mathcal{T}^{K}\right)$. By Corollary 19.8 in [1] of the non-linear Peetre theorem we can assume that $B$ is a polynomial (of arbitrary degree). The regularity of $D$ implies that

$$
\left\langle D(B)_{\Theta}, \frac{d}{d t_{0}}\left(t j_{(0,0)}^{p, p, p}\left(x^{i}, X^{I}, 0, \ldots, 0,1,0, \ldots, 0\right)\right) \otimes \frac{\partial}{\partial Y^{2}}{ }_{0}\right\rangle
$$

is smooth with respect to the coordinates of $B$. Then by the homogeneous function theorem (and the above type of homogeneity) we deduce that

$$
\left\langle D(B)_{\Theta}, \frac{d}{d t_{0}}\left(t j_{(0,0)}^{p, p, p}\left(x^{i}, X^{I}, 0, \ldots, 0,1,0, \ldots, 0\right)\right) \otimes \frac{\partial}{\partial Y^{2}}{ }_{0}\right\rangle
$$

depends linearly on the coordinates of $B$ on all $x^{\varrho} X^{\sigma} Y_{(\beta, \gamma)}^{1} d Y^{2} \otimes d^{m_{1}} x \wedge$ $d^{m_{2}} X$ and $x^{\varrho} X^{\sigma} Y_{(\beta, \gamma)}^{2} d Y^{1} \otimes d^{m_{1}} x \wedge d^{m_{2}} X$, it depends bilinearly on the coordinates of $B$ on all $x^{\varrho} X^{\sigma} d Y^{1} \otimes d^{m_{1}} x \wedge d^{m_{2}} X$ and $x^{\varrho} X^{\sigma} d Y^{2} \otimes d^{m_{1}} x \wedge$ $d^{m_{2}} X$, and it is independent of the other coordinates of $B$, where (of course) $\left(x^{i}, X^{I}, y_{\alpha}^{k}, Y_{(\beta, \gamma)}^{K}\right)$ is the induced coordinate system on the prolongation $J^{r, s, q}\left(\mathbb{R}^{m_{1}, m_{2}, n_{1}, n_{2}}\right)$ and $d^{m_{1}} x=d x^{1} \wedge \cdots \wedge d x^{m_{1}}$ and $d^{m_{2}} X=d X^{1} \wedge \cdots \wedge$ $d X^{m_{2}}$. (Here and in what follows, $\alpha, \beta$ are arbitrary $m_{1}$-tuples and $\gamma$ is an arbitrary $m_{2}$-tuple with $|\alpha| \leq q,|\beta|+|\gamma| \leq r$ or $|\gamma| \leq s$ if $\left.\beta=(0)\right)$.

In other words (and more precisely),

$$
\left\langle D(B)_{\Theta}, \frac{d}{d t_{0}}\left(t j_{(0,0)}^{r, s, q}\left(x^{i}, X^{I}, 0, \ldots, 0,1,0, \ldots, 0\right)\right) \otimes \frac{\partial}{\partial Y^{2}}{ }_{0}\right\rangle
$$

is determined by the values

$$
\begin{aligned}
& \left\langle D\left(x^{\varrho} X^{\sigma} Y_{(\beta, \gamma)}^{2} d Y^{1} \otimes d^{m_{1}} x \wedge d^{m_{2}} X\right)_{\Theta},\right. \\
& \left.\frac{d}{d t_{0}}\left(t j_{(0,0)}^{r, s, q}\left(x^{i}, X^{I}, 0, \ldots, 0,1,0, \ldots, 0\right)\right) \otimes \frac{\partial}{\partial Y^{2}}{ }_{0}\right\rangle, \\
& \left\langle D\left(x^{\varrho} X^{\sigma} Y_{(\beta, \gamma)}^{1} d Y^{2} \otimes d^{m_{1}} x \wedge d^{m_{2}} X\right)_{\Theta},\right. \\
& \left.\frac{d}{d t}{ }_{0}\left(t j_{(0,0)}^{r, s, q}\left(x^{i}, X^{I}, 0, \ldots, 0,1,0, \ldots, 0\right)\right) \otimes \frac{\partial}{\partial Y^{2}} 0\right\rangle,
\end{aligned}
$$




$$
\begin{aligned}
& \left\langle D\left(x^{\varrho} X^{\sigma} d Y^{1} \otimes d^{m_{1}} x \wedge d^{m_{2}} X+x^{\widetilde{\varrho}} X^{\widetilde{\sigma}} d Y^{2} \otimes d^{m_{1}} x \wedge d^{m_{2}} X\right)_{\Theta},\right. \\
& \frac{d}{d t_{0}}\left(t j_{(0,0)}^{r, s, q}\left(x^{i}, X^{I}, 0, \ldots, 0,1,0, \ldots, 0\right)\right) \otimes \frac{\partial}{\partial Y^{2}} 0 .
\end{aligned}
$$

Furthermore, $\left\langle D(B)_{\Theta}, \frac{d}{d t}\left(t j_{(0,0)}^{r, s, q}\left(x^{i}, X^{I}, 0, \ldots, 0,1,0, \ldots, 0\right)\right) \otimes \frac{\partial}{\partial Y^{2}}{ }_{0}\right\rangle$ is linear in $B$ for $B$ from the $\mathbb{R}$-vector subspace spanned by all elements $x^{\varrho} X^{\sigma} Y_{(\beta, \gamma)}^{1} d Y^{2} \otimes d^{m_{1}} x \wedge d^{m_{2}} X$ and $x^{\widetilde{\varrho}} X^{\widetilde{\sigma}} Y_{(\beta, \gamma)}^{2} d Y^{1} \otimes d^{m_{1}} x \wedge d^{m_{2}} X$; moreover,

$$
\begin{array}{r}
\left\langle D\left(d Y^{1} \otimes d^{m_{1}} x \wedge d^{m_{2}} X+B\right)_{\Theta},\right. \\
\left.\quad \frac{d}{d t_{0}}\left(t j_{(0,0)}^{r, s, q}\left(x^{i}, X^{I}, 0, \ldots, 0,1,0, \ldots, 0\right)\right) \otimes \frac{\partial}{\partial Y^{2}}{ }_{0}\right\rangle \\
=\left\langle D(B)_{\Theta}, \frac{d}{d t_{0}}\left(t j_{(0,0)}^{r, s, q}\left(x^{i}, X^{I}, 0, \ldots, 0,1,0, \ldots, 0\right)\right) \otimes \frac{\partial}{\partial Y^{2}}{ }_{0}\right\rangle
\end{array}
$$

for $B$ from the vector subspace (over $\mathbb{R}$ ) spanned by all $x^{\varrho} X^{\sigma} Y_{(\beta, \gamma)}^{1} d Y^{2} \otimes$ $d^{m_{1}} x \wedge d^{m_{2}} X$ and $x^{\widetilde{\varrho}} X^{\widetilde{\sigma}} Y_{(\beta, \gamma)}^{2} d Y^{1} \otimes d^{m_{1}} x \wedge d^{m_{2}} X$; and

$$
\begin{array}{r}
\left\langle D\left(a x^{\varrho} X^{\sigma} d Y^{1} \otimes d^{m_{1}} x \wedge d^{m_{2}} X+b x^{\widetilde{\varrho}} X^{\widetilde{\sigma}} d Y^{2} \otimes d^{m_{1}} x \wedge d^{m_{2}} X\right)_{\Theta},\right. \\
\left.\frac{d}{d t_{0}}\left(t_{(0,0)}^{r, s, q}\left(x^{i}, X^{I}, 0, \ldots, 0,1,0, \ldots, 0\right)\right) \otimes \frac{\partial}{\partial Y^{2}}{ }_{0}\right\rangle \\
=a b\left\langle D\left(x^{\varrho} X^{\sigma} d Y^{1} \otimes d^{m_{1}} x \wedge d^{m_{2}} X+x^{\widetilde{\varrho}} X^{\widetilde{\sigma}} d Y^{2} \otimes d^{m_{1}} x \wedge d^{m_{2}} X\right)_{\Theta},\right. \\
\left.\frac{d}{d t}\left(t j_{(0,0)}^{r, s, q}\left(x^{i}, X^{I}, 0, \ldots, 0,1,0, \ldots, 0\right)\right) \otimes \frac{\partial}{\partial Y^{2}}{ }_{0}\right\rangle
\end{array}
$$

for all real numbers $a$ and $b$.

Then by the invariance of $D$ with respect to $\left(\tau^{i} x^{i}, \mathcal{T}^{I} X^{I}, y^{k}, Y^{K}\right)$ for $\tau^{i} \neq 0$ and $\mathcal{T}^{I} \neq 0$ we get

$$
\begin{aligned}
& \left\langle D\left(x^{\varrho} X^{\sigma} Y_{(\beta, \gamma)}^{2} d Y^{1} \otimes d^{m_{1}} x \wedge d^{m_{2}} X\right)_{\Theta},\right. \\
& \left.\frac{d}{d t_{0}}\left(t j_{(0,0)}^{r, s, q}\left(x^{i}, X^{I}, 0, \ldots, 0,1,0, \ldots, 0\right)\right) \otimes \frac{\partial}{\partial Y^{2}}{ }_{0}\right\rangle \\
& =\left\langle D\left(x^{\varrho} X^{\sigma} Y_{(\beta, \gamma)}^{1} d Y^{2} \otimes d^{m_{1}} x \wedge d^{m_{2}} X\right)_{\Theta},\right. \\
& \left.\frac{d}{d t}\left(t j_{(0,0)}^{r, s, q}\left(x^{i}, X^{I}, 0, \ldots, 0,1,0, \ldots, 0\right)\right) \otimes \frac{\partial}{\partial Y^{2}}{ }_{0}\right\rangle=0
\end{aligned}
$$


for $(\beta, \gamma) \neq(\varrho, \sigma)$, and

$$
\begin{aligned}
\left\langleD \left( x^{\varrho} X^{\sigma} d Y^{1} \otimes d^{m_{1}} x\right.\right. & \left.\wedge d^{m_{2}} X+x^{\widetilde{\varrho}} X^{\widetilde{\sigma}} d Y^{2} \otimes d^{m_{1}} x \wedge d^{m_{2}} X\right)_{\Theta}, \\
& \left.\frac{d}{d t_{0}}\left(t j_{(0,0)}^{r, s, q}\left(x^{i}, X^{I}, 0, \ldots, 0,1,0, \ldots, 0\right)\right) \otimes \frac{\partial}{\partial Y^{2}}{ }_{0}\right\rangle=0
\end{aligned}
$$

for all $\varrho, \widetilde{\varrho}, \sigma$ and $\widetilde{\sigma}$.

Hence $D$ is determined by the evaluations

$$
\begin{aligned}
& \left\langle D\left(x^{\beta} X^{\gamma} Y_{(\beta, \gamma)}^{1} d Y^{2} \otimes d^{m_{1}} x \wedge d^{m_{2}} X\right)_{\Theta},\right. \\
& \left.\frac{d}{d t_{0}}\left(t j_{(0,0)}^{r, s, q}\left(x^{i}, X^{I}, 0, \ldots, 0,1,0, \ldots, 0\right)\right) \otimes \frac{\partial}{\partial Y_{20}}\right\rangle, \\
& \left\langle D\left(x^{\beta} X^{\gamma} Y_{(\beta, \gamma)}^{2} d Y^{1} \otimes d^{m_{1}} x \wedge d^{m_{2}} X\right)_{\Theta},\right. \\
& \left.\frac{d}{d t_{0}}\left(t j_{(0,0)}^{r, s, q}\left(x^{i}, X^{I}, 0, \ldots, 0,1, \ldots, 0\right)\right) \otimes \frac{\partial}{\partial Y^{2}}{ }_{0}\right\rangle .
\end{aligned}
$$

Suppose $\beta^{i_{0}} \neq 0$ for some $i_{0}=1, \ldots, m_{1}$. We use the invariance of $D$ with respect to the locally defined $\mathcal{F} \mathcal{M}_{m_{1}, m_{2}, n_{1}, n_{2}}$-map

$$
\psi^{i_{0}}=\left(x^{i}, X^{I}, y^{k}, Y^{1}, Y^{2}+x^{i_{0}} Y^{2}, Y^{3}, \ldots, Y^{n_{2}}\right)^{-1}
$$

preserving $x^{i}, X^{I},, \Theta, Y^{1}, j_{(0,0)}^{r, s, q}\left(x^{i}, X^{I}, 0, \ldots, 0,1,0, \ldots, 0\right), \frac{\partial}{\partial Y^{2}} 0$ and sending $Y_{(\beta, \gamma)}^{2}$ to $Y_{(\beta, \gamma)}^{2}+x^{i_{0}} Y_{(\beta, \gamma)}^{2}+Y_{\left(\beta-1_{i}, \gamma\right)}^{2}$. Applying this invariance to

$$
\begin{aligned}
\left\langleD \left( x^{\beta-1_{i}} X^{\gamma} Y_{(\beta, \gamma)}^{2} d Y^{1} \otimes d^{m_{1}} x\right.\right. & \left.\wedge d^{m_{2}} X\right)_{\Theta}, \\
& \left.\frac{d}{d t_{0}}\left(t j_{(0,0)}^{r, s, q}\left(x^{i}, X^{I}, 0, \ldots, 0,1,0, \ldots, 0\right)\right) \otimes \frac{\partial}{\partial Y^{2}}{ }_{0}\right\rangle
\end{aligned}
$$

it follows that the value (3) is zero if it is zero for $\beta-1_{i_{0}}$ instead of $\beta$. Continuing this process and a similar one for the $\mathcal{F} \mathcal{M}_{m_{1}, m_{2}, n_{1}, n_{2}}$-morphism

$$
\Psi^{I_{0}}=\left(x^{i}, X^{I}, y^{k}, Y^{1}, Y^{2}+X^{I_{0}} Y^{2}, Y^{3}, \ldots, Y^{n_{0}}\right)^{-1}
$$

instead of $\psi^{i_{0}}$ we see that (3) is zero if it is zero for $(\beta, \gamma)=((0),(0))$.

By similar arguments (since $\psi^{i_{0}}$ sends $d Y^{2}$ to $d Y^{2}+x^{i_{0}} d Y^{2}$ and $\Psi^{I_{0}}$ sends $d Y^{2}$ to $d Y^{2}+X^{I_{0}} d Y^{2}$ ), from the equality

$$
\begin{aligned}
\left\langleD \left( x^{\beta-1_{i}} X^{\gamma} Y_{(\beta, \gamma)}^{1} d Y^{2} \otimes d^{m_{1}} x\right.\right. & \left.\wedge d^{m_{2}} X\right)_{\Theta}, \\
& \left.\frac{d}{d t}\left(t j_{(0,0)}^{r, s, q}\left(x^{i}, X^{I}, 0, \ldots, 0,1,0, \ldots, 0\right)\right) \otimes \frac{\partial}{\partial Y^{2}}{ }_{0}\right\rangle=0
\end{aligned}
$$


for $\beta_{i_{0}} \neq 0$ (or a similar equality for $\gamma_{I_{0}} \neq 0$ ) we find that $(2)$ is zero if $(\beta, \gamma) \neq((0),(0))$.

In other words, $D$ is uniquely determined by the values (2) and (3) for $(\beta, \gamma)=((0),(0))$.

Using the invariance of $D$ with respect to the (local) $\mathcal{F} \mathcal{M}_{m_{1}, m_{2}, n_{1}, n_{2}}$-map

$$
\left(x^{i}, X^{I}, y^{k}, Y^{1}+Y^{1} Y^{2}, Y^{2}, \ldots, Y^{n_{1}}\right)^{-1}
$$

preserving $\Theta, j_{(0,0)}^{r, s, q}\left(x^{i}, X^{I}, 0, \ldots, 0,1,0, \ldots, 0\right)$ and $\frac{\partial}{\partial Y^{2}} 0$, from the equality

$$
\begin{aligned}
\left\langleD \left( d Y^{1} \otimes d^{m_{1}} x\right.\right. & \left.\wedge d^{m_{2}} X\right)_{\Theta}, \\
& \left.\frac{d}{d t_{0}}\left(t j_{(0,0)}^{r, s, q}\left(x^{i}, X^{I}, 0, \ldots, 0,1,0, \ldots, 0\right)\right) \otimes \frac{\partial}{\partial Y^{2}}{ }_{0}\right\rangle=0
\end{aligned}
$$

(see (1)) we deduce that

$$
\begin{aligned}
& \left\langle D\left(Y_{((0),(0))}^{2} d Y^{1} \otimes d^{m_{1}} x \wedge d^{m_{2}} X\right)_{\Theta},\right. \\
& \left.\frac{d}{d t_{0}}\left(t j_{(0,0)}^{r, s, q}\left(x^{i}, X^{I}, 0, \ldots, 0,1,0, \ldots, 0\right)\right) \otimes \frac{\partial}{\partial Y^{2}}{ }_{0}\right\rangle \\
& =-\left\langle D\left(Y_{((0),(0))}^{1} d Y^{2} \otimes d^{m_{1}} x \wedge d^{m_{2}} X\right)_{\Theta},\right. \\
& \left.\frac{d}{d t_{0}}\left(t j_{(0,0)}^{r, s, q}\left(x^{i}, X^{I}, 0, \ldots, 0,1,0, \ldots, 0\right)\right) \otimes \frac{\partial}{\partial Y^{2}}{ }_{0}\right\rangle .
\end{aligned}
$$

Thus $D$ is uniquely determined by

$$
\begin{aligned}
& \left\langle D\left(Y_{((0),(0))}^{2} d Y^{1} \otimes d^{m_{1}} x \wedge d^{m_{2}} X\right)_{\Theta},\right. \\
& \left.\quad \frac{d}{d t_{0}}\left(t j_{(0,0)}^{r, s, q}\left(x^{i}, X^{I}, 0, \ldots, 0,1,0, \ldots, 0\right)\right) \otimes \frac{\partial}{\partial Y^{2}}{ }_{0}\right\rangle \in \Lambda^{m} T_{0}^{*} \mathbb{R}^{m}=\mathbb{R} .
\end{aligned}
$$

So the vector space of all $D$ in question is of dimension less than or equal to 1 . Hence $D=c \mathcal{H}$ for some $c \in \mathbb{R}$.

\section{References}

[1] I. Kolár̆, Natural operations related with the variational calculus, in: Differential Geometry and its Applications (Opava, 1992), Silesian Univ. Opava, 1993, 461-472.

[2] I. Kolář, P. W. Michor and J. Slovák, Natural Operations in Differential Geometry, Springer, Berlin, 1993.

[3] I. Kolář and R. Vitolo, On the Helmholtz operator for Euler morphisms, Math. Proc. Cambridge Philos. Soc. 135 (2003), 277-290.

[4] W. M. Mikulski, On naturality of the Helmholtz operator, Arch. Math. (Brno) 41 (2005), 145-149. 
[5] W. M. Mikulski, The jet prolongations of fibered fibered manifolds and the flow operator, Publ. Math. Debrecen 59 (2001), 441-458.

[6] - On the variational calculus in fibered-fibered manifolds, Ann. Polon. Math. 89 (2006), 1-12.

[7] R. Wolak, On transverse structures on foliations, Suppl. Rend. Circ. Mat. Palermo 9 (1985), 227-243.

Institute of Mathematics

Jagiellonian University

Reymonta 4

30-059 Kraków, Poland

E-mail: mikulski@im.uj.edu.pl

Received 7.4.2006

and in final form 24.8.2006 